\title{
Response to Bryan Roberts: A new perspective on $T$ violation
}

\author{
Abhay Ashtekar* \\ Institute for Gravitation and the Cosmos 89 Physics Department, \\ Penn State, University Park, PA 16802, U.S.A.
}

\begin{abstract}
It is surprising that the fundamental, microscopic laws of Nature are not invariant under time reversal. In his article, Three Merry Roads to T-Violation, Dr. Bryan Roberts provided a succinct summary of the theoretical frameworks normally used to interpret the results of the experiments that established this fact. They all rely on the detailed structure of quantum mechanics. In this 'response' to Dr. Robert's talk, I will show that these experiments can be interpreted using a much more general framework. Consequently, should quantum mechanics be eventually replaced by a new paradigm, e.g., because of quantum gravity, these experiments could still be used to argue that the microscopic laws violate $T$ invariance.
\end{abstract}

\section{PREAMBLE}

Dr. Roberts has provided a lucid account [1] of the conceptual arguments that have been used to show that the fundamental, microscopic laws of Nature fail to be invariant under the time-reversal operation, $T$. His clear presentation led me to sharpen my own understanding of the theoretical constructs that have been used to analyze experiments in this area. This response is the result of that re-examination. I have attempted to make it reasonably selfcontained but, to fully appreciate its content, it would be helpful if the reader has already gone through Dr. Robert's paper.

I will focus on the two approaches to T-violation - referred to as the Curie and the Kabir principles in [1] and recalled below - that have already been used to analyze experiments. However, following this workshop, Dr. Roberts has also extended the third approach, based on Wigner's non-degeneracy principle, to the general setting presented in this article [2]. ${ }^{1}$

To interpret any experiment, one needs a theoretical paradigm. For T-violation experiments, the standard choice has been the mathematical framework of quantum physics. This is natural because the best available description of the electroweak interactions is based on local quantum field theory. However, in this response, I will show that one can in fact analyze the $T$-violation experiments in a much more general setting. Classical mechanics, quantum mechanics and quantum field theory provide only specific examples of this general framework. As is often the case, because this framework has much less structure, the analysis becomes significantly simpler. As a result, aspects of quantum physics that are truly

\footnotetext{
*Electronic address: ashtekar@gravity.psu.edu

1 Perhaps the most promising experiments to realize this idea are the measurements of the electric dipole moment of elementary particles, such as neutrons. However, the dipole moment fails to be invariant also under the parity operation $P$. Therefore, even if one were to observe a non-zero electric dipole moment, one would need to cleanly remove the parity contribution before one can draw definitive conclusions about $T$ violation. Consequently this route to experimentally demonstrating $T$-violation has been quite difficult.
} 
essential for interpreting experiments on $C P$ and $T$-violation are brought to the forefront. They are clearly separated from other features which, though not central, have generally been treated in the literature as being equally significant. More importantly, the conceptual structure that is essential to the interpretation of experiments is so weak that, even if quantum physics were to be replaced by some more general framework - e.g., because of its unification with general relativity - the current experiments will still, in all likelihood, enable us to conclude that the fundamental laws of Nature fail to be invariant under time reversal.

The issue of time-reversal has two distinct facets: microscopic and macroscopic. The microscopic $T$-violation discussed in [1] and in the present communication is quite distinct from the manifest arrow of time we perceive in our everyday life and, more generally, in the physics of large systems. Since Dr. Roberts mentioned the macro-world only in passing, before entering the main discussion, let me begin with a brief detour to explain this point. For simplicity, I will use the framework of classical physics because the core of the argument is not sensitive to the distinction between classical and quantum mechanics. Consider a large box with a partition that divides it into two parts, say, the right and the left halves. Suppose there is some gas in the left half and vacuum in the right. Once the equilibrium is reached, the macroscopic state of this gas is described by the volume it occupies, $V_{i}$; the pressure it exerts on the walls of the box, $P_{i}$; and its temperature $T_{i}$; where $i$ stands for 'initial'. If we open the partition slowly, the gas will fill the whole box and its macro-state in equilibrium will be described by new parameters, $V_{f}, P_{f}, T_{f}$. Thus, there has been a transition from the initial macro-state $\left(V_{i}, P_{i}, T_{i}\right)$ to a final state $\left(V_{f}, P_{f}, T_{f}\right)$. Our common experience tells us that the time reverse of this process is extremely unlikely. However, we also know that the microscopic variables for the system are the positions and momenta of some $10^{23}$ molecules in the box. These are subject just to Newton's laws which are manifestly invariant under the time-reversal operation T! Therefore, if we were to reverse the momentum $\vec{p}_{(\alpha)}(t)$ of each molecule (labeled by $\alpha$ ) at a late time $t$, keeping the positions $\vec{x}_{(\alpha)}(t)$ the same, time evolution would indeed shift the gas back from its final macroscopic state to the initial one, confined just to the left half of the box. But in practice it is very difficult to construct this timereversed initial state. Thus, there is indeed a macroscopic arrow of time but its origin is not in the failure of the microscopic laws to be invariant under $T$ but rather in the fact that the initial conditions we normally encounter are very special. Specifically, in our example, there are vastly fewer micro-states compatible with the initial macro-state $\left(V_{i}, P_{i}, T_{i}\right)$ than those that are compatible with the final macro-state $\left(V_{f}, P_{f}, T_{f}\right){ }^{2}$ Put differently, the entropy of the initial macro-state is much lower than that in the final macro-state. This is the arrow of time, commonly discussed in the literature.

It is clear from the simple example that the fact that there is an obvious arrow of time in the macro-world does not imply that the microscopic or fundamental laws have to break $T$-invariance. Indeed, as Dr. Roberts emphasized in the beginning of his article [1], it was common to assume that the fundamental laws are invariant under the time-reversal operation $T$. It came as a shock that the weak force violates this 'self-evident' premise!

The rest of this article will focus on the $T$ violation at the fundamental, microscopic level.

\footnotetext{
${ }^{2}$ This is primarily because the volume $V_{f}$ allowed for each molecule in the final macro-state is twice as large as $V_{i}$, allowed in the initial macro-state. Consequently, the number of microscopic configurations compatible with the final macroscopic state is about $2^{10^{23}}$ times the number of microscopic states compatible with the initial macroscopic configuration.
} 


\section{WEAK INTERACTIONS AND THE CURIE PRINCIPLE}

As Dr. Roberts has explained, what the Cronin-Fitch experiment [3] establishes directly is that the weak interactions are not invariant under $C P$, i.e., under the simultaneous operations of charge conjugation $C$ and reflection through a mirror, $P$. As normally formulated, the parity operation is meaningful only if the underlying space-time is flat, i.e., represented by Minkowski space-time. This means one ignores curvature and therefore gravity. One further assumes that physics is described by a local quantum field theory on this Minkowski space, for which individual physical fields transform covariantly under the action of the Lorentz group, and dynamics is generated by a self-adjoint Hamiltonian obtained by integrating a scalar density (or a 3-form), constructed locally from the physical fields. Then, one has the CPT theorem that guarantees that the product $C P T$ of charge conjugation, $C$, parity, $P$ and time reversal $T$ is an exact dynamical symmetry. ${ }^{3}$ Therefore, although the Cronin-Fitch experiment does not directly imply $T$-violation, as Dr. Roberts explained, if we assume that weak interactions are described by a local quantum field theory in Minkowski space, then the observed breakdown of $\mathrm{CP}$ invariance implies that they violate $T$ invariance as well. In the rest of this section, I will focus on just the $C P$ symmetry and its violation observed in the Cronin-Fitch experiment. Thus, I will not need to refer to the CPT at all.

In the current analysis of $C P$ violation, one uses the following form of the Curie principle: If an initial state $\sigma_{i}$ is in variant under $C P$ but its time-evolved final state $\sigma_{f}$ is not, then dynamics cannot be $C P$ invariant. As explained in section 2.5 of [1], the analysis has the remarkable feature that it does not assume a specific Hamiltonian $H$. Therefore, the argument will remain unchanged should we discover that the currently used Hamiltonian $H$ in electro-weak interactions has to be modified, e.g., to accommodate future experiments, or to unify them with strong interactions.

However, the standard analysis does make a crucial use of the detailed kinematical structure of quantum physics (summarized below). If a future quantum gravity theory were to require that this structure has to be modified - e.g., by removing the emphasis on linearitythen the standard analysis cannot be used to conclude that the Cronin-Fitch experiment implies a violation of $C P$ invariance in weak interactions. The main point of this section is to show that this specific kinematical framework of Hilbert spaces and operators is not really necessary. The Curie principle can be extended to a much more general framework than that offered by quantum physics.

Let us begin by introducing this framework, which we will call general mechanics. It will incorporate quantum as well as classical mechanics, but only as specific special cases. The basic assumptions of general mechanics are:

- i) We have a set $\mathcal{S}$ of states;

\footnotetext{
3 This is a summary of the discussion one finds in quantum field theory text books (see, e.g. [4]). More rigorous versions based on Weightman axioms [5] and the algebraic approach [6] are also available in the literature. However, the self-adjoint Hamiltonian required in the text book version is not rigorously defined in four dimensions beyond the quantum theory of free fields. Similarly, we still do not have a single example of a 4-dimensional, interacting quantum field theory satisfying either the Wightman axioms or the axioms of the algebraic quantum field theory. Thus, there is a curious mis-match between the mathematical statements of CPT theorems and theories of direct physical interest.
} 
- ii) There is a 1-1, onto dynamical map $S$ - the ' $S$-matrix' - from $\mathcal{S}$ to itself. This $S$ could refer to finite time evolution, say from time $t_{1}$ to $t_{2}$ or, alternatively, to the time evolution in the infinite past to the infinite future. In practice is it convenient to consider two copies $\mathcal{S}_{i}$ and $\mathcal{S}_{f}$ of $\mathcal{S}$, representing initial and final states, and regard $S$ as a map from $\mathcal{S}_{i}$ to $\mathcal{S}_{f}$

$$
S: \mathcal{S}_{i} \rightarrow \mathcal{S}_{f} ; \quad S\left(\sigma_{i}\right)=\sigma_{f} \quad \forall \sigma_{i} \in \mathcal{S}_{i}
$$

- iii) Potential symmetries are represented by a 1-1, onto map $R: \mathcal{S} \rightarrow \mathcal{S}$, from $\mathcal{S}$ to itself. We will first consider the case in which $R$ maps $\mathcal{S}_{i}$ to itself and $\mathcal{S}_{f}$ to itself. This is the case if $R$ is, for example, the discrete symmetry represented by $C$, or $P$ or $C P$.

Note that the framework is truly minimalistic. The space $\mathcal{S}$ of states is just a set; no further structure is needed. To draw the contrast, let me summarize the structure we use in classical and quantum mechanics. ${ }^{4}$ In classical mechanics, $\mathcal{S}$ is the phase space which is assumed to be a smooth, even dimensional differentiable manifold $\Gamma$, equipped with a non-degenerate, closed 2-form $\Omega$, called the symplectic structure. In quantum mechanics, one first introduces an auxiliary structure, a Hilbert space, $H$ : it is a complex vector space that is endowed with an Hermitian scalar product and is complete in the sense that the limit point of each Cauchy sequence in $H$ is again in $H$. Pure states are then represented by rays in $H$ and mixed states by density matrices, i.e., bounded, self-adjoint operators with finite trace. As for dynamics, in classical mechanics, it is given by the flow generated by a Hamiltonian vector field $\left.X=\Omega^{-1}\right\urcorner d H$ for a smooth function $H$ on $\Gamma$, called the Hamiltonian. Now the map $S$ corresponds either to the finite $S$-matrix between a time $t_{1}$ and $t_{2}$, obtained by moving along the Hamiltonian flow an affine parameter distance $t_{2}-t_{1}$, or its limit as $t_{1} \rightarrow-\infty$ and $t_{2} \rightarrow \infty$. In quantum mechanics, as mentioned above, dynamics is represented by a unitary flow $\mathcal{U}_{t}=\exp -i t H$ generated by a self-adjoint operator. The map $S$ corresponds again either to a finite $S$ matrix, $\mathcal{U}_{t_{2}-t_{1}}$, or its limit as $t_{1} \rightarrow-\infty$ and $t_{2} \rightarrow \infty$. Finally, in classical mechanics the potential symmetries $R$ are diffeomorphisms on $\Gamma$ that preserve the symplectic structure $\Omega$ and those in quantum mechanics are linear, unitary mappings on $H$.

This rather detailed discussion serves to brings out three points. First, both classical and quantum mechanics are based on very rich but specific and intricate mathematical frameworks. From the summary of the literature contained in section 2.4 of [1], it would appear that the specifics of quantum mechanics - the Hilbert space structure of $\mathcal{H}$ and the linearity of the candidate symmetries $R$ - are all essential for a formulation of the Curie principle that can be used to interpret the Cronin-Fitch experiment. Second, the mathematical underpinnings of the classical and the quantum frameworks are very different from each other. Therefore, it is natural to anticipate that the next paradigm shift (that may emerge, e.g., from a successful quantum gravity theory) could bring with it another rich yet different mathematical framework. Would one then have to reconstruct an appropriate formulation of the Curie principle and re-examine experiments using the new framework? The third point is that this would not be necessary if one could develop a formulation of the

\footnotetext{
${ }^{4}$ But the material till the end of this paragraph can be skipped without loss of continuity. It brings to forefront the intricacy of the structure underlying classical and quantum mechanics, in contrast to general mechanics.
} 
Curie principle (and also the Kabir principle, discussed in section III) using just the three assumptions of general mechanics which have survived the transition from classical physics to quantum. Indeed, the assumptions are so minimalistic that it is reasonable to hope that they will also be realized in the future, more general mechanics.

Let us then investigate if the Curie principle can be formulated using just the three assumptions of general mechanics. In the spirit of this principle, suppose that there exists some initial state $\sigma_{i} \in \mathcal{S}$ such that

$$
R \sigma_{i}=\sigma_{i} \quad \text { but } \quad R \sigma_{f} \neq \sigma_{f}
$$

Then,

$$
R\left(S \sigma_{i}\right)=R \sigma_{f} \neq \sigma_{f}, \quad \text { but } \quad \sigma_{f}=S\left(\sigma_{i}\right)=S\left(R \sigma_{i}\right) .
$$

Therefore, we conclude

$$
S R \neq R S .
$$

Thus, the dynamical map $S$ does not commute with the candidate symmetry $R: \quad R$ can not a dynamical symmetry. To summarize, we have derived the Kabir principle in the framework of general mechanics: If there exists a state $\sigma_{i}$ such that $R \sigma_{i}=\sigma_{i}$ and $S \sigma_{i}=\sigma_{f}$ but $R \sigma_{f} \neq \sigma_{f}$ then $R$ is not a dynamical symmetry of the system. ${ }^{5}$ By repeating the argument of [1], now it is easy to analyze the Cronin-Fitch experiment in the setting of general mechanics. Even if the theory describing weak interaction is more general than quantum mechanics, the experiment would still imply that the dynamics of weak interactions violates $T$-invariance, so long as the underlying framework of this theory meets the three basic postulates of general mechanics.

Note that this general formulation in particular enables one to discuss in one go all the symmetries $R$ in classical mechanics and linear symmetries used in quantum mechanics. Furthermore, since one does not even need to refer to the underlying Hilbert space structure, one can also apply the argument within standard quantum mechanics even if $R$ or $S$ were represented by non-linear mappings. In particular, for the argument to hold, the $C P$ symmetry does not have to be unitarily realized. By contrast, as summarized in [1], the current literature on the Cronin-Fitch experiment often emphasizes that the assumption of unitarity is essential to conclude that the experiment implies $C P$ violation.

We will conclude this discussion of the Curie principle with three remarks.

1) Since $\mathcal{S}$ is only a set, we cannot speak of continuous evolution in the framework introduced above. But one could trivially extend the framework by endowing $\mathcal{S}$ with a topology and replacing $S$ with a continuous evolution map $E(t)$, where $t$ is to be thought of as a time parameter. The argument given above will then imply that $E(t)$ will not commute with $R$.

2) However, we did assume that $R$ maps the space $\mathcal{S}_{i}$ of initial states to itself and the space $\mathcal{S}_{f}$ of final states to itself. This assumption is not satisfied by the time-reversal operation $T$, which maps initial states to final states (and vice versa): $T: \mathcal{S}_{i} \rightarrow \mathcal{S}_{f}$ is a 1-1 onto map from $S_{i}$ to $S_{f}$. Therefore, in this case, $T$ is a dynamical symmetry if and only if

$$
S \sigma_{i}=\sigma_{f} \quad \Longrightarrow \quad S^{-1}\left(T \sigma_{i}\right)=T^{-1}\left(\sigma_{f}\right)=T^{-1}\left(S \sigma_{i}\right)
$$

\footnotetext{
${ }^{5}$ It is straightforward to alter the argument to obtain the other desired conclusion of fact 1 in section 2.4 of [1].
} 
i.e., the time reverse of $\sigma_{i}$ (which is in $\mathcal{S}_{f}$ ) is mapped by dynamics to the time reverse of $\sigma_{f}$. The generalization of the Curie principle discussed above does not have any implication in this case. Therefore to directly test $T$-invariance we need a generalization of the Kabir principle, discussed in section III. In this respect, the situation is the same as in section 2 of $[1]$.

3) While $R$ invariance of dynamics is captured by the property $R S=S R$ of the S-matrix $S$, the $T$ invariance is captured by $S^{-1} T=T^{-1} S$. As we just saw, the difference arises simply because, while $R$ preserves each of $\mathcal{S}_{i}$ and $\mathcal{S}_{f}, T$ maps one to the other. At a fundamental level, then, the difference is not because $R$ is linear while $T$ is anti-linear, as is often stated in the literature: If one works entirely in the quantum framework, one misses the broader perspective and is then tempted to focus only on the anti-linearity of $T$.

\section{THE KABIR PRINCIPLE}

By using the Curie principle in conjunction with the Cronin-Fitch experiment we have concluded that $C P$ cannot be a dynamical symmetry in any theory of weak interactions that conforms to the principles of general mechanics. But this provides only an indirect evidence for $T$-violation because one needs to assume, in addition, validity of the $C P T$ theorem. As explained in section 3 of [1], more direct evidence for $T$-violation comes from another idea, called the Kabir principle by Roberts, which is again formulated in the framework of quantum mechanics: If a transition $\sigma_{i} \rightarrow \sigma_{f}$ occurs with a different probability from the transition $T \sigma_{f} \rightarrow T \sigma_{i}$ then the dynamics underlying this process cannot be invariant under the time-reversal operation, T. Again this argument is deeply rooted in the detailed structure of quantum mechanics. A natural question for us is then: Can we extend the Kabir principle to general mechanics? The answer is in the affirmative. However, since it refers to probabilities, even to state Kabir's principle one needs to introduce some additional structure in general mechanics.

Let us then introduce the necessary structure, in addition to the three postulates stated in section II:

- An overlap map $\mathcal{O}$ on the space of states $\mathcal{S}$ (and therefore on each of $\mathcal{S}_{i}$ and $\mathcal{S}_{f}$ ): $\mathcal{O}: \mathcal{S} \times \mathcal{S} \rightarrow[0,1] \in R$, such that

$$
\mathcal{O}\left(\sigma, \sigma^{\prime}\right)=\mathcal{O}\left(\sigma^{\prime}, \sigma\right), \quad \forall \sigma, \sigma^{\prime} \in \mathcal{S} .
$$

$\mathcal{O}\left(\sigma, \sigma^{\prime}\right)$ is to be thought of as the overlap between states $\sigma$ and $\sigma^{\prime}$.

In classical mechanics, general states are represented by non-negative distributions $\rho$ on the phase space $\Gamma$ which are normalized so that $\int_{\Gamma} \rho d V_{\Omega}=1$, where $d V_{\Omega}$ is the Liouville volume element on $\Gamma$. Then the overlap function $\mathcal{O}\left(\rho, \rho^{\prime}\right)$ can be the obvious overlap between the two states: $\mathcal{O}\left(\rho, \rho^{\prime}\right)=\int_{\Gamma}\left(\rho \rho^{\prime}\right)^{\frac{1}{2}} d V_{\Omega}{ }^{6}$ In quantum mechanics, a general state is represented by a normalized density matrix $\hat{\rho}$-a non-negative, bounded, self-adjoint operator on the

\footnotetext{
${ }^{6}$ The square-root in the integrand ensures that the overlap function is bounded by 1 . (It is not needed in quantum theory.) A pure state $\rho=\rho_{\gamma}$, is just the Dirac distribution peaked at a point $\gamma$ of $\Gamma$. For these states, natural regularizations yield : $\mathcal{O}\left(\rho_{\gamma}, \rho_{\gamma^{\prime}}\right)=\delta_{\gamma, \gamma^{\prime}}$. Thus the overlap between a pure state and a distinct pure state simply vanishes while its overlap with itself is perfect.
} 
Hilbert space $\mathcal{H}$ with $\operatorname{Tr} \hat{\rho}=1$, where $\operatorname{Tr}$ is the trace operation. The overlap function can be then taken to be $\mathcal{O}\left(\hat{\rho}, \hat{\rho}^{\prime}\right)=\operatorname{Tr} \hat{\rho} \hat{\rho}^{\prime}$. Pure states - projection operators on one dimensional subspaces of $\mathcal{H}$ - can be represented by (equivalence classes of) normalized elements $\psi$ of $\mathcal{H}$ (where two elements are equivalent if they differ by a phase). Then the overlap function $\mathcal{O}\left(\psi, \psi^{\prime}\right)$ is simply the standard overlap probability of quantum mechanics: $\mathcal{O}\left(\psi, \psi^{\prime}\right)=\left|\left\langle\psi, \psi^{\prime}\right\rangle\right|^{2}$.

Let us return to general mechanics. Since the overlap map is part of the kinematical structure, the notion of symmetries and dynamics, introduced in section II, have to be compatible with it. The compatibility conditions are all natural and obvious. But since they will be used in the proof of the Kabir principle, let us spell them out explicitly.

- To qualify as symmetry, the map $R: \mathcal{S}_{i} \rightarrow \mathcal{S}_{i}$ introduced in section II has to satisfy

$$
\mathcal{O}_{i}\left(R \sigma_{i}, R \sigma_{i}^{\prime}\right)=\mathcal{O}_{i}\left(\sigma_{i}, \sigma_{i}^{\prime}\right)
$$

on $\mathcal{S}_{i}$ (and similarly on $\mathcal{S}_{f}$ ). Similarly to qualify as symmetry, the time reversal map $T: \mathcal{S}_{i} \rightarrow \mathcal{S}_{f}$ must satisfy

$$
\mathcal{O}_{f}\left(T \sigma_{i}, T \sigma_{i}^{\prime}\right)=\mathcal{O}_{i}\left(\sigma_{i}, \sigma_{i}^{\prime}\right)
$$

for all $\sigma_{i} \in \mathcal{S}_{i}$.

- The dynamical map $S: \mathcal{S}_{i} \rightarrow \mathcal{S}_{f}$ should be compatible with the overlap map, i.e., satisfy

$$
\mathcal{O}_{i}\left(\sigma_{i}, \sigma_{i}^{\prime}\right)=\mathcal{O}_{f}\left(S \sigma_{i}, S \sigma_{i}^{\prime}\right) \equiv \mathcal{O}_{f}\left(\sigma_{f}, \sigma_{f}^{\prime}\right) .
$$

Finally, using the overlap map $\mathcal{O}$ and the dynamical map $S$, we introduce the notion of transition probability:

- Given a dynamical map $S$, the transition probability between an initial state $\sigma_{i} \in \mathcal{S}_{i}$ and any given final state $\sigma_{f}^{\prime}$ is defined to be

$$
P\left(\sigma_{f}^{\prime}, \sigma_{i}\right):=\mathcal{O}_{f}\left(\sigma_{f}^{\prime}, S \sigma_{i}\right) \equiv \mathcal{O}_{f}\left(\sigma_{f}^{\prime}, \sigma_{f}\right) .
$$

This concludes the additional kinematical structure we need on our general mechanics to formulate the Kabir principle. Note that the notions of the overlap function and transition probability are already available in classical as well as quantum mechanics. In both cases symmetries and dynamics satisfy the compatibility conditions we have introduced. Thus, as in section II, general mechanics continues to be minimalist: it simply extracts certain notions that are common to both classical and quantum paradigms, which we can reasonably expect to be present also in the future generalizations of these settings.

Recall that $T$ is a dynamical symmetry if and only if $S^{-1} T=T^{-1} S$, or, equivalently, $S T^{-1}=T S^{-1}$. Suppose a dynamical map $S$ satisfies this condition. As before, let us use the notation $\sigma_{f}=S \sigma_{i}$ and $\sigma_{f}^{\prime}=S \sigma_{i}^{\prime}$. Then,

$$
\mathcal{O}_{f}\left(T \sigma_{i}, S\left(T^{-1} \sigma_{f}^{\prime}\right)\right)=\mathcal{O}_{f}\left(T \sigma_{i}, T\left(S^{-1} \sigma_{f}^{\prime}\right)\right)=\mathcal{O}_{f}\left(T \sigma_{i}, T \sigma_{i}^{\prime}\right)=\mathcal{O}_{i}\left(\sigma_{i}, \sigma_{i}^{\prime}\right)
$$

where in the last step we used (3.3). On the other hand, (3.4) and (3.1) imply

$$
\mathcal{O}_{i}\left(\sigma_{i}, \sigma_{i}^{\prime}\right)=\mathcal{O}_{f}\left(S \sigma_{i}, S \sigma_{i}^{\prime}\right)=\mathcal{O}_{f}\left(S \sigma_{i}^{\prime}, S \sigma_{i}\right)=\mathcal{O}_{f}\left(\sigma_{f}^{\prime}, S \sigma_{i}\right) .
$$


The last two equations and the definition (3.5) of transition probability now yield

$$
P\left(\sigma_{f}^{\prime}, \sigma_{i}\right)=P\left(T \sigma_{i}, T^{-1} \sigma_{f}^{\prime}\right) .
$$

Thus, we have shown that if $T$ is a symmetry of the dynamical map $S$ then the transition probability between the states $\sigma_{i}$ and $\sigma_{f}^{\prime}$ must equal that between the two states $T^{-1} \sigma_{f}^{\prime}$ and $T \sigma_{i}$, obtained by a time-reversal. Therefore if the transition probability between any two states and their time reversed versions differ observationally, then the time reversal symmetry is broken by dynamics. ${ }^{7}$ This is the generalization of the Kabir principle from quantum mechanics to general mechanics we were seeking. As Dr. Roberts has explained, it has been used to show that the kaon oscillations, $K^{0} \leftrightarrows \bar{K}^{0}$ observed at the CPLEAR detector provide a direct violation of $T$-invariance ( see, e.g., [7-9]). Using the same arguments as in section 3.2 of [1], we can now conclude that the CPLEAR observations imply that the time-reversal symmetry $T$ is violated in any theory of weak interactions so long as it falls in the broad framework of general mechanics developed in this section.

As with the discussion of the generalized Curie principle of section II, this generalization of the Kabir criterion does not refer to the Hilbert space structure of the space of states or linearity (or anti-linearity) of various maps. In particular, in the case of quantum mechanics, while it incorporates the standard treatment neatly summarized in section 3 of [1], the result would hold even if, say, the S-matrix were anti-unitary (or, indeed, non-linear!). By contrast, as emphasized in [1], it is generally believed that unitarity of the $S$ matrix is essential to conclude that we have experimental evidence of direct $T$-violation in weak interactions.

\section{DISCUSSION}

Already in the context of quantum mechanics, it is interesting to analyze the interplay between symmetries and dynamics using only that structure which is available in general mechanics. The analysis becomes much simpler and, furthermore, brings to forefront the concepts and structures that are essential in the discussions of $C P$ and $T$ violations, separating them from inessential notions that have added unnecessary complexity to the analyses one finds in the literature. In particular, neither the linear structure nor the details of the Hermitian inner product on the space of quantum mechanical states is essential. Nor is it necessary for symmetries to be represented by unitary or anti-unitary operators; indeed, they need not even be linear operators! Secondly, the primary distinction between $T$ on the one hand, and $C, P$, or $C P$ on the other, is only that while the spaces of 'in' and 'out' states are separately invariant under $C, P$ and $C P$, the map $T$ sends the incoming states to the outgoing ones. In standard quantum mechanics, $C, P$ and $C P$ are represented by linear, unitary maps, while $T$ is represented by an anti-linear, anti-unitary map. However, if one uses only that structure which is provided by general mechanics one finds that, contrary to a wide-spread belief, this difference is not primary to the distinction between the Curie and the Kabir criteria.

\footnotetext{
${ }^{7}$ It is worth noting that, in practice, one measures is the transition rate. This is not determined solely by the transition probability. In the leading order approximation ("Fermi's golden rule") the transition probability has to be multiplied by the density of final states. But in practice one can easily take care of this issue and verify whether or not the transition probability is symmetric under time-reversal.
} 
While these points are illuminating, the main strength of the more general perspective presented here is that it broadens the reach of the Cronin-Fitch and CPLEAR experiments enormously. We are no longer constrained to analyze them in the strict framework of quantum mechanics. This is a significant advantage because future advances, particularly in quantum gravity, may well lead us to a mathematical framework which is quite different from that offered by quantum mechanics. Just as in the passage from special relativity to general relativity we had to abandon flatness of space-time geometry, the future theory may well force us to abandon the linear structure that underlies quantum mechanics. For example, in quantum mechanics the pure states are represented by rays in the Hilbert space $\mathcal{H}$ and constitute a trivial Kähler manifold. It may be replaced by general, non-trivial Kähler manifolds which do not refer to a Hilbert space. Or, dynamics and symmetries may be represented by non-linear maps. Indeed, there are concrete ideas as to how this may come about (see, e.g., [10] and references therein). But such generalizations are likely to retain the minimal structure of general mechanics, introduced in sections II and III. If so, then the current experiments will still continue to imply that the dynamics of weak interactions violates $C P$ and $T$ invariance even in these much more general theories. Thus, at a conceptual level the reach of the current experiments on $C P$ and $T$ violations seems to be extraordinarily long, going well beyond the standard quantum mechanics framework in which they have generally been interpreted.

To conclude, I would like to return to a broader issue of time reversal asymmetry, beyond the violations observed in weak interactions. In section I, I began with a discussion of the distinction between the macroscopic and microscopic arrows of time. As I explained there, the macroscopic arrow can arise even when the microscopic laws are manifestly timesymmetric because the initial conditions we normally encounter are very special. In physical sciences the focus is generally on laws of Nature and initial conditions are considered less fundamental as they can and do vary from one experiment to another and, furthermore, are influenced by the environment. But in the spirit of this workshop, let us consider the cosmological setting. One is then led to stipulate that the macroscopic arrow of time arises because the initial conditions of the universe are very special. But then the goal of science should also include the development of a theory of these initial conditions. At a cosmological level, the distinction between the fundamental laws and initial conditions could well become blurry, or disappear altogether, and there may be new, fundamental laws that severely constrain the initial conditions, leading to the gross violation of time reversal symmetry in the macroscopic phenomena. ${ }^{8}$ These laws will likely violate not only $T$ invariance but even the CPT invariance that underlies quantum field theory in Minkowski space-time [11].

\section{Acknowledgments}

I would like to thank Bryan Roberts for stimulating discussions, John Collins for a valuable e-mail exchange and Emily Grosholz, John Norton and Tom Pashby for raising insight-

\footnotetext{
${ }^{8}$ In particular, within general relativity, Penrose [11] has proposed a Weyl curvature hypothesis that says that the initial singularities are very special in that the Weyl curvature vanishes there (while the Ricci curvature diverges). Gell-Mann, Hartle, Hawking and others (see e.g. [12]) have made other proposals starting from a generalization of the quantum mechanics of closed systems. More recently, the two sets of ideas have begun to distill in a coherent setup in the framework of loop quantum cosmology [13].
} 
ful questions that have improved this presentation. Additional thanks are due to Emily Grosholz for her leadership in bringing together physicists and philosophers for a lively workshop. This work was supported in part by the NSF grant PHY-1205388 and the Eberly research funds of Penn state.

[1] B. W. Roberts, Three merry roads to T-violation, talk at the Workshop on Cosmologogy and Time, held at Penn State, April 2013 (pre-print); http:/ philsci-archive.pitt.edu/9632; http:/xxx.lanl.gov/abs/1306.6382.

[2] B. W. Roberts, Comment on Ashtekar: Generalization of Wigner's Principle, 2013 (pre-print); http:/xxx.lanl.gov/abs/1306.6381.

[3] J. H.Christenson, J. W. Cronin, V. L. Fitch and R. Turlay, Evidence for the $2 \pi$ decay in the $K_{2}^{0}$ meson, Physical Review Letters, 13, 138-140 (1964).

[4] S. Weinberg, Quantum Theory of Fields Vol 1, (Cambridge University Press, Cambridge, 1995), pp 244-246.

[5] R. F. Streater and A. Wightman, PCT, Spin, Statistics and All That, Chapter 4 (Benjamin, New York, 1964).

[6] J. Yngvason and H. J. Borchers, On the PCT-theorem in the theory of local observables, arXiv:math-ph/0012020.

[7] CPLEAR collaboration, First direct observation of time reversal non-invariance in the neutral kaon system, Physics Letters, B444, 43-51 (1998).

[8] L. Alvarez-Gaume, C. Kounnas and P. Pavlopoulos, Violation of time reversal invariance and CPLEAR measurements, Physics Letters, B458, 347-354 (1999).

[9] J. Ellis and N. E. Mavromatos, Comments on CP, T and CPT violation in neutral koan decays, Physics Reports, 320, 341-354, (1999).

[10] A. Ashtekar and T. Schilling, Geometrical formulation of quantum mechanics, in: On Einsteins Path, edited by A. Harvey (Springer-Verlag, Berlin, 1998), pp 23-66; also available at arXiv:gr-qc/9706069.

[11] R. Penrose, Singularities and time-asymmetry, in General relativity: An Einstein centenary survey, edited by S.W. Hawling and W. Israel (Cambridge University Press, Cambridge, 1979).

[12] J. B. Hartle, Spacetime Quantum Mechanics and the Quantum Mechanics of Spacetime, in Gravitation and Quantizations: Proceedings of the 1992 Les Houches Summer School edited by by B. Julia and J. Zinn-Justin (North Holland, Amsterdam, 1995).

[13] I. Agullo, A. Ashtekar and W. Nelson, The pre-inflationary dynamics of loop quantum cosmology: Confronting quantum gravity with observations, Classical and Quantum Gravity, 30 085014 (2013). 